\title{
Atomic Scale Deformation Mechanisms of Amorphous Polyethylene under Tensile Loading
}

\author{
M.A. Tschopp ${ }^{1}$, J.L. Bouvard ${ }^{1}$, D.K. Ward $^{2}$, M.F. Horstemeyer ${ }^{1}$ \\ ${ }^{1}$ Center for Advanced Vehicular Systems, Mississippi State University; Starkville, MS 39759, USA \\ ${ }^{2}$ Sandia National Laboratory; Livermore, CA 94551, USA
}

Keywords: Molecular Dynamics, Polyethylene, Deformation

\begin{abstract}
The molecular origin of plastic deformation within amorphous polymers is an important issue that is still not well understood. Here, we investigate the deformation mechanisms of amorphous polyethylene. Molecular dynamics simulations were used to apply a tensile load to amorphous polyethylene using a united atom model. A range of strain rates, chain lengths and chain numbers were sampled to investigate the stress-strain behavior and associated deformation mechanisms. The large range in these conditions (e.g., 100 times difference in strain rate) provided the contrast in response necessary to elucidate the relationship between intrachain (Van der Waal's interactions) and interchain (dihedral rotations) deformation mechanisms in amorphous polyethylene. Simulation results show how internal mechanisms associated with chain rotation and entanglement evolve with increasing deformation. These results provide insight into the evolution of internal state variables for multiscale models of thermoplastic polymers.
\end{abstract}

\section{Introduction}

The molecular origin of plastic deformation within amorphous polymers is an important issue that is still not well understood. Atomistic simulations have provided insight into the plastic deformation behavior of some thermoplastic polymer systems. However, although several groups have studied the static, dynamic, and mechanical properties of polyethylene (PE) systems, research on large systems, particularly for mechanical properties, is still in its infancy. Molecular dynamics (MD) and molecular statics simulations have proven to be essential tools for exploring the static, dynamic and mechanical properties of bulk amorphous polymers at the molecular levels. The objective of this research is to study the relevant deformation mechanisms responsible for the different regimes (elasticity/yield/strain softening/strain hardening) observed during uniaxial tension deformation of glassy PE using atomic-scale simulations with a united atom model. Here, we have investigated the stress-strain response and the energy partitioning evolution for different chain lengths, number of chains and strain rates. In addition to stressstrain response, we also evaluated internal mechanisms associated with bond length, bond angle, dihedral distributions, free volume and chain entanglements; for brevity, we present only chain rotation and entanglements here. Our main interest was correlating the stress-strain response to the deformation mechanisms responsible for the mechanical behavior.

\section{Simulation Methodology}

Interatomic potential

The interatomic force field for amorphous PE is based on a united atom model using the DREIDING potential [1]. There were several reasons why this potential was chosen. First, this study investigates uniaxial deformation of amorphous PE systems with large numbers of monomers (up to 200,000 united atoms) as a function of strain rate. Full atom representations would require approximately three times the number of atoms $\left(\mathrm{CH}_{2}\right)$ along with a more computationally-expensive force field and, quite possibly, a smaller timestep or different integration scheme due to adding hydrogen. Therefore, the use of the united atom model and DREIDING potential was influenced in part by the computational expense. Second, much of the prior literature regarding MD simulations of PE has used the DREIDING potential. In this sense, this work can build upon results previously obtained, while also providing guidance as to the influence of boundary conditions when moving to full atom representations with optimized force fields. We leave deformation of full-atom polymer representations for future work.

The united atom model used here considers each methyl group (i.e., the $\mathrm{CH}_{2}$ monomer) as a single atom with a force field that determines the interactions between united atoms. The DREIDING potential [1] has four contributing terms; bond stretching, changes in bond angle, changes in dihedral rotation, and van der Waals non- 
bonded interactions. The total force field energy can be expressed as

$$
E_{\text {total }}=E_{b}(r)+E_{\theta}(\theta)+E_{\phi}(\phi)+E_{n b}(r)
$$

In the present simulations, the interaction potential has two components which are summed over all possible atom combinations: bonded and non-bonded interaction terms. The bonded terms comprise bond stretching (r), bondangle bending $(\theta)$ and dihedral angle torsion $(\phi)$. The functional form of bonded energy is given as

$$
\begin{aligned}
& E_{b}(r)=\frac{1}{2} K_{b}\left(r-r_{0}\right)^{2} \\
& E_{\theta}(\theta)=\frac{1}{2} K_{\theta}\left(\theta-\theta_{0}\right)^{2} \\
& E_{\phi}(\phi)=\sum_{i=0}^{3} C_{i}(\cos \phi)^{i}
\end{aligned}
$$

where $K_{b}$ and $K_{\theta}$ are the stiffness constants for the bond length and bond angle potentials, respectively, $r_{0}$ and $\theta_{0}$ are the equilibrium bond length and bond angle, respectively, and the variable $C_{i}$ contains the coefficients of dihedral multi-harmonic. For the parameters chosen in the present work, the global minimum of the dihedral potential is at $180^{\circ}$, which corresponds to the planar trans state. In addition, the two local gauche minima are located at $60^{\circ}$ and $300^{\circ}$. The non-bonded or Van der Waals interactions are given by a Lennard-Jones potential, i.e.,

$$
E_{n b}(r)=4 \epsilon\left[\left(\frac{\sigma}{r}\right)^{12}-\left(\frac{\sigma}{r}\right)^{6}\right], r \leq r_{c}
$$

where $r$ is the distance between two atoms, $\sigma$ is the distance at zero energy, and $\epsilon$ is the energy well depth. The cutoff distance $r_{c}$ is taken as 10.5 Angstroms. The parameters for the PE force field are given in the Table 1.

\section{Deformation simulations}

A parallel MD code, LAMMPS [2], that incorporates domain decomposition was used to deform the polymer simulation cells. A Nosé-Hoover thermostat was used to regulate the system temperature $[3 ; 4]$. The simulations were performed for three chain lengths $(100,1000$, and 2000 monomers) with three different number of chains (20, 200 and 2000) for a total of $2 \times 10^{4}$ to $2 \times 10^{5}$ total united atoms; little effect was found with respect to chain length and chain number. The mechanical responses for each simulation resulted from averages of three different initial configurations to account for entropic effects due to the high number of possible chain conformations. Temperatures of
$100 \mathrm{~K}$ and $250 \mathrm{~K}$ were examined here to select a temperature sufficiently below the glass transition temperature, $T_{g}$, and one close to room temperature but just below $T_{g}$. The initial chain structure was created using a method similar to those previously developed using Monte Carlo self-avoiding random walks [5]. The chain generation step used a face-centered cubic (FCC) lattice superimposed on the simulation cell with the nearest neighbor distance of 1.53 Angstroms. Molecules were added to the lattice in a probabilistic stepwise manner, which based the probability of chain growth in certain directions on the bond angle and the density of unoccupied sites in the region.

This initial structure was then inserted into the MD code where an equilibration sequence was performed prior to deforming the amorphous polymer. The equilibration sequence relaxes any high energy configurations that are artificially created due to the FCC lattice used to generate the amorphous polymer structure. The relaxation involves four different steps. Initially, the simulation ran for $10^{5}$ timesteps $(\Delta \mathrm{t}=1 \mathrm{fs})$ using NVT dynamics at 500 $\mathrm{K}$ followed by relaxation for $5 \times 10^{5}$ timesteps $(\Delta \mathrm{t}=0.5$ fs) using NPT dynamics at $500 \mathrm{~K}$. The next relaxation cooled the structure down to the desired temperature for $5 \times 10^{5}$ timesteps followed by further relaxation of $5 \times 10^{5}$ timesteps $(\Delta \mathrm{t}=1 \mathrm{fs})$ at the desired temperature $(100 \mathrm{~K}$ and $250 \mathrm{~K})$.

Several microstructure metrics were used to quantitatively validate the polymer chain geometry following equilibration. The average bond length for all equilibrated systems was 1.529 Angstroms and the average bond angle was $109.27^{\circ}$. The average values were slightly lower than the equilibrium potential parameters $r_{0}$ and $\theta_{0}$. The dihedral angle distribution has a broad distribution with both gauche and trans peaks. To calculate the fraction of trans conformations within the PE system, a threshold value of $120^{\circ}$ was used to delineate the gauche peak (cen-

\begin{tabular}{cc}
\hline Parameters & Values \\
\hline$K_{b}$ & $350 \mathrm{kcal} / \mathrm{mol}$ \\
$r_{0}$ & $1.53 \mathrm{Angstroms}$ \\
$K_{\theta}$ & $60 \mathrm{kcal} / \mathrm{mol} / \mathrm{rad}^{2}$ \\
$\theta_{0}$ & $1.911 \mathrm{rad}$ \\
$C_{0}$ & $1.736 \mathrm{kcal} / \mathrm{mol}$ \\
$C_{1}$ & $-4.490 \mathrm{kcal} / \mathrm{mol}$ \\
$C_{2}$ & $0.776 \mathrm{kcal} / \mathrm{mol}$ \\
$C_{3}$ & $6.990 \mathrm{kcal} / \mathrm{mol}$ \\
$\sigma$ & $4.01 \mathrm{Angstroms}$ \\
$\epsilon$ & $0.112 \mathrm{kcal} / \mathrm{mol}$ \\
\hline
\end{tabular}

Table 1: Interatomic potential parameters 
tered about $66^{\circ}$ ) from the trans peak (centered about $\left.180^{\circ}\right)$. The percent trans conformations in the initial structure was $73.8 \%$ and $68.3 \%$ for the $100 \mathrm{~K}$ and 250 $\mathrm{K}$ samples, respectively. The initial densities for the amorphous PE structures ranged from $0.87-0.91 \mathrm{~g} / \mathrm{cm}^{3}$, slightly lower than experimental values for low density PE (0.91-0.94 g/ $\left.\mathrm{cm}^{3}\right)$, which contains a high degree of short and long chain branching. The glass transition temperature was used to verify that such equilibration of the polymer structure was appropriate for deformation simulations. The glass transition temperature $T_{g}$, delimiting the glassy regime to the rubbery one, was determined from the change in slope of the specific volume vs. temperature curve. The calculated values of $\approx 300 \mathrm{~K}$ here are similar to those calculated by other groups using $\mathrm{MD}$, which fall in the range of $250-300 \mathrm{~K}$, close to the experimentally-measured value of $250 \mathrm{~K}$.

The amorphous PE system was then deformed under a uniaxial tensile strain applied at a constant strain rate with a zero-pressure condition for the two lateral simulation cell faces. This deformation condition was implemented in LAMMPS by decoupling the boundary in the loading direction from the NPT equations of motion [6]. The stress components were calculated from the symmetric pressure tensor, which uses components from the kinetic energy tensor and the virial tensor. In addition to calculating the stress as a function of strain, the energy contributions associated with the bond length, bond angles, dihedral angle and non-bonding interactions were tracked as a function of strain. Periodic dumps of the atomic configuration were performed to compute additional microstructure metrics such as chain orientation or the percentage of trans dihedral conformations. Three different strain rates $\left(10^{8} \mathrm{~s}^{-1}, 10^{9} \mathrm{~s}^{-1}\right.$ and $\left.10^{10} \mathrm{~s}^{-1}\right)$ were used with an initial configuration consisting of 20 polymeric chains, each having 1000 monomers, to investigate the effect of strain rate. To investigate the influence of chain length and chain number, PE configurations were uniaxially deformed at a strain rate of $10^{9} \mathrm{~s}^{-1}$ for different numbers of chains (100 and 2000) and chain lengths (20, 200, and 2000 monomers). The procedure used here and the results shown within are described in more detail in Hossain et al. [7].

\section{Simulation Results}

Stress-strain response

The stress-strain response was averaged for multiple configurations and the isothermal stress-strain curve for a 20-chain, 1000-monomer per chain amorphous PE system

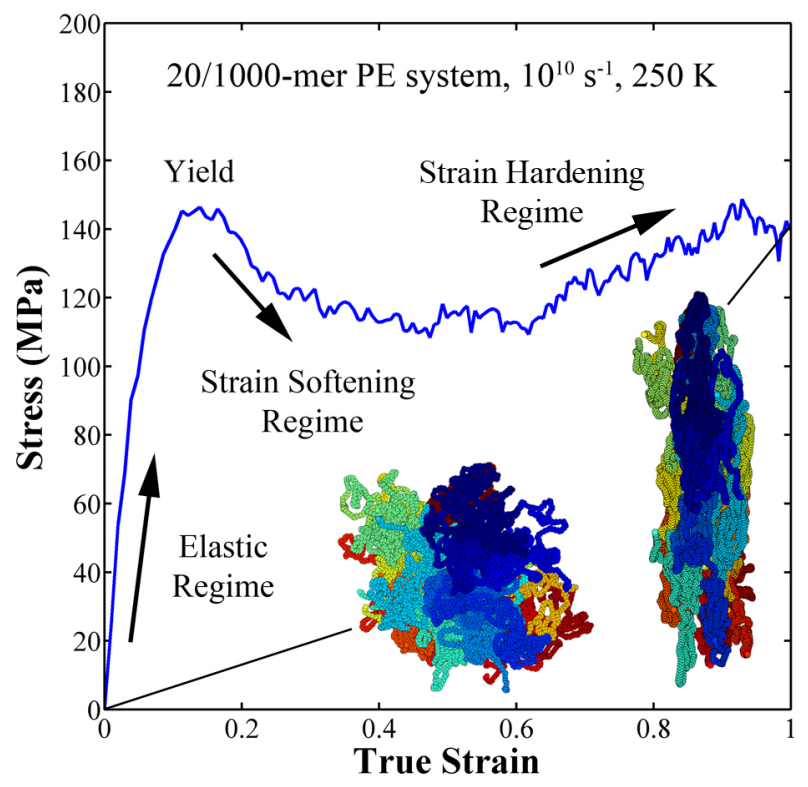

Figure 1: Stress-strain response of amorphous polyethylene deformed in uniaxial tension at strain rate of $10^{10}$ $\mathrm{s}^{-1}$ and temperature of $250 \mathrm{~K}$. The accompanying images show the polyethylene structure after equilibration and at $100 \%$ true strain (colors represent united atoms on separate chains).

deformed at $250 \mathrm{~K}$ and $10^{10} \mathrm{~s}^{-1}$ strain rate is shown in Figure 1. The stress-strain curve has four distinct regimes typical of experimental curves: elastic, yield, softening and hardening. Initially, in the elastic regime, the stress increases nearly linearly with increasing applied strain. Interestingly, the characteristic yield peak observed in experiments is also observed at strain rates of $10^{10} \mathrm{~s}^{-1}$, but is not observed at lower strain rates. Upon reaching the yield point, the stress then shows a decrease in stress for the strain softening regime. Further deformation of PE causes an increase in stress during the strain hardening regime.

Figure 2 shows the stress-strain curves for a 20-chain, 1000-monomer per chain amorphous PE system deformed at $250 \mathrm{~K}$ using strain rates of $10^{8} \mathrm{~s}^{-1}, 10^{9} \mathrm{~s}^{-1}$ and $10^{10}$ $\mathrm{s}^{-1}$. The simulations at different strain rates exhibit different stress-strain curves. The stress-strain curve at a strain rate of $10^{10} \mathrm{~s}^{-1}$ follows the typical trend with an elastic regime, followed by yield, and then followed by a strain softening and strain hardening regime, as shown in Fig. 1. The elastic modulus and peak yield stress increases with increasing strain rate. The trend of higher stresses for higher strain rates continues for the strain 


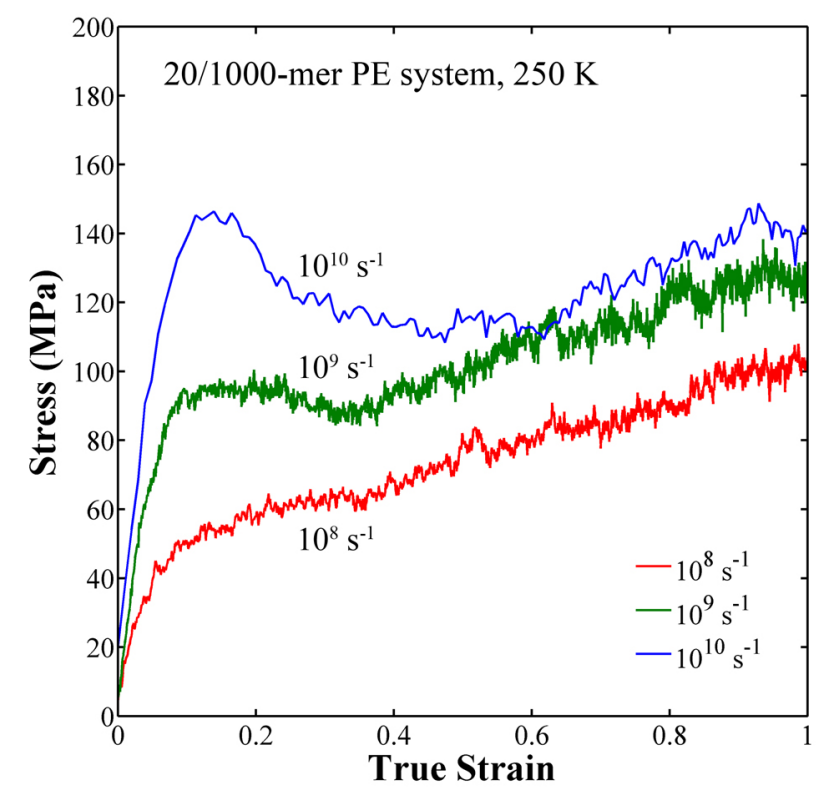

Figure 2: Stress-strain response at $250 \mathrm{~K}$ for different strain rates.

softening and strain hardening regimes as well. However, the constant strain hardening slope indicates that the mechanisms may be similar between the different strain rates. The elastic modulus and the yield stress increase with increasing strain rate and decreasing temperature.

\section{Internal energy evolution}

Figure 3(a) shows the potential energy change of a 20 chain, 1000-monomer per chain amorphous PE system deformed at $250 \mathrm{~K}$ for a strain rate of $10^{10} \mathrm{~s}^{-1}$, which corresponds to the stress-strain behavior observed in Figure 1. In addition to the total energy $E_{\text {total }}$, the individual components of the energy are shown: the non-bonded energy $E_{n b}$, the bonded energy $E_{b}$, the bond angle energy $E_{\theta}$ and the dihedral energy $E_{\phi}$. In the elastic region, the dihedral, angle and bond energies remain almost unchanged and a majority of the increase in total energy is correlated with an increase in the non-bonded energy, i.e., Van der Waal's forces between polymer chains. The non-bonded energy increases sharply in the elastic and strain softening regions, which can be associated with chain slippage mechanisms. After the elastic region, the non-bonded energy stays fairly constant while there are significant changes in the bond angle and dihedral energies. The energy associated with bond length steadily decreases as bond lengths move towards the equilibrium bond length. The dihedral angle energy increases in the strain softening regime and then steadily decreases in the strain hardening regime as gauche conformations rotate to the lower energy trans conformations. The total energy sharply increases in the

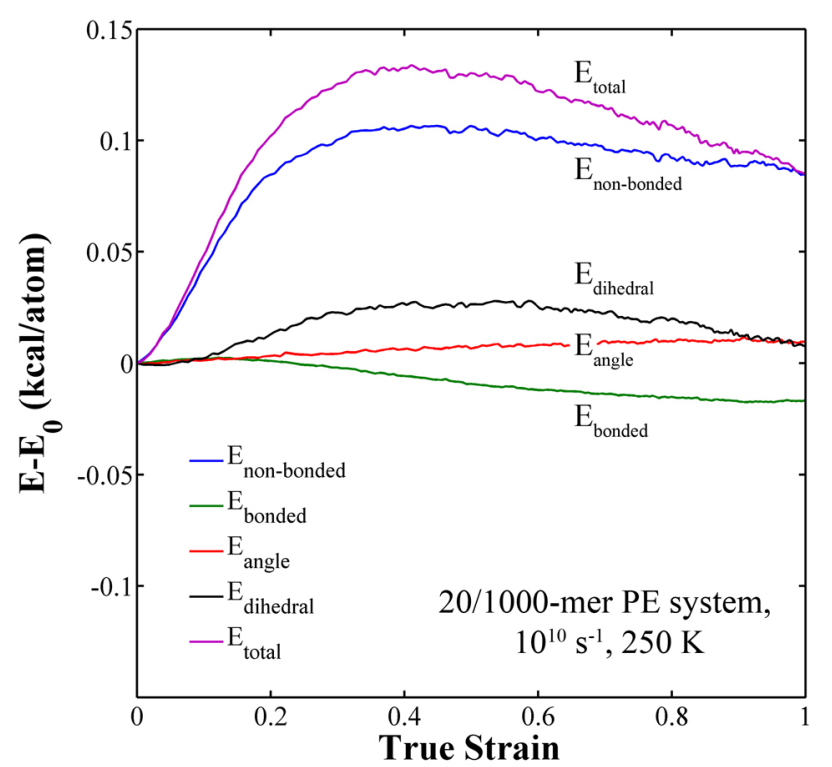

(a)

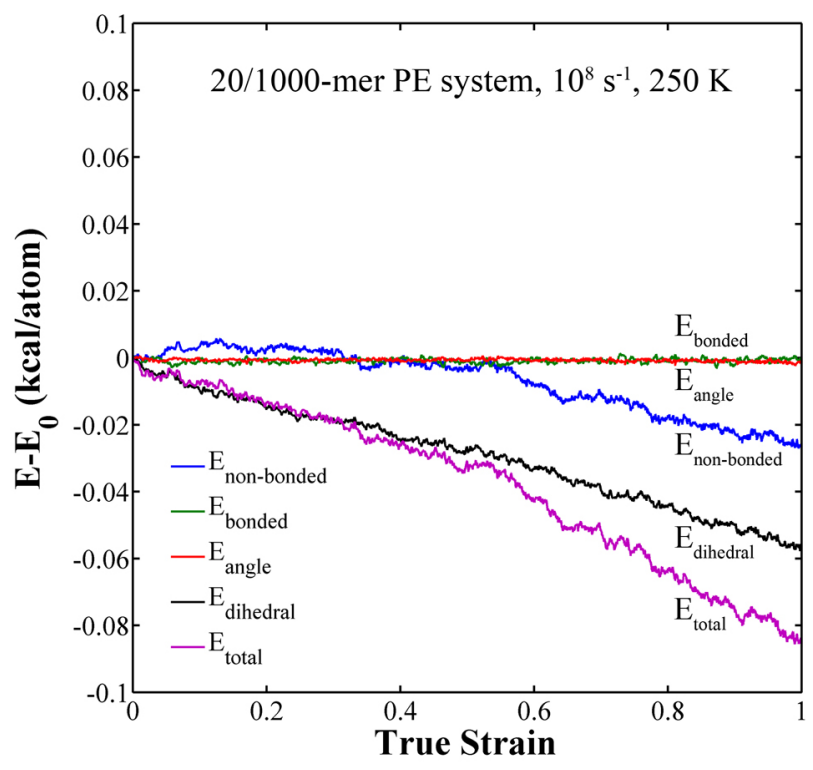

(b)

Figure 3: Energy decomposition for a polyethylene (PE) system with 20 chains consisting of 1000 monomers each at $250 \mathrm{~K}$ for a strain rate of $10^{10} \mathrm{~s}^{-1}$. The non-bonded energy changes most during the elastic regime, while the dihedral and bonded energy changes most during the strain softening/hardening regimes. 
elastic region due to non-bonded interactions and then slowly decreases over the strain softening and hardening regions as energy is dissipated via dihedral rotations and bond length changes to accommodate deformation.

Figure 3(b) shows the variation in internal energy for a strain rate of $10^{8} \mathrm{~s}^{-1}$ for a 20-chain, 1000-monomer per chain amorphous PE system at $250 \mathrm{~K}$. The lower strain rate has a significant effect on the energy evolution. Note the decrease in the non-bonded energy as strain rate is lowered. In fact, for a strain rate of $10^{8} \mathrm{~s}^{-1}$, there is very little change in the energies associated with bond length and bond angles with increasing strain. The non-bonded interaction energy only slightly increases before decreasing with increasing strain. The dihedral angle (and total) energy immediately decreases with increasing strain during the elastic region, unlike the behavior observed for $10^{10} \mathrm{~s}^{-1}$ strain rate in Fig. 3. Interestingly, because the dihedral energy decrease occurs during the elastic regime at a strain rate of $10^{8} \mathrm{~s}^{-1}$, the non-bonded interaction energy does not increase as sharply as higher strain rates, signifying a change in where the energy associated with deformation is accommodated at lower strain rates.

\section{$\underline{\text { Internal structure evolution }}$}

The change in the dihedral distribution from gauche to trans conformations as a function of strain is also important to deformation in polymer systems. Figure 4 shows how the \%trans conformations evolves as a function of strain at $250 \mathrm{~K}$. The \%trans conformations in the simulation cell was calculated using a dihedral angle threshold of $120^{\circ}$. Additionally, this data is plotted for three different strain rates and for two different chain length and chain number combinations (20 1000monomer chains, 2000 100-monomer chains) to compare behavior between different conditions. First, the \%trans conformations increases at a faster rate as the strain rate decreases, i.e., a strain rate of $10^{8} \mathrm{~s}^{-1}$ has the fastest increase in trans conformations. This is as expected; the lower strain rate will allow more time for chains to rotate to trans conformations. However, the main difference between the three strain rates occurs in the elastic and strain softening regimes $\left(\epsilon_{\text {true }}<0.5\right)$, and the increase in \%trans conformations during the strain hardening region is nearly constant. Notice that the $10^{10} \mathrm{~s}^{-1}$ strain rate shows very little increase in the \%trans conformations for the elastic region, in agreement with previous simulations and experiments. The effect of the polymer chain length was also examined and one representative curve is included in Figure 4 for comparison. For a strain rate of $10^{9} \mathrm{~s}^{-1}$, the 2000 100-monomer chain PE system shows

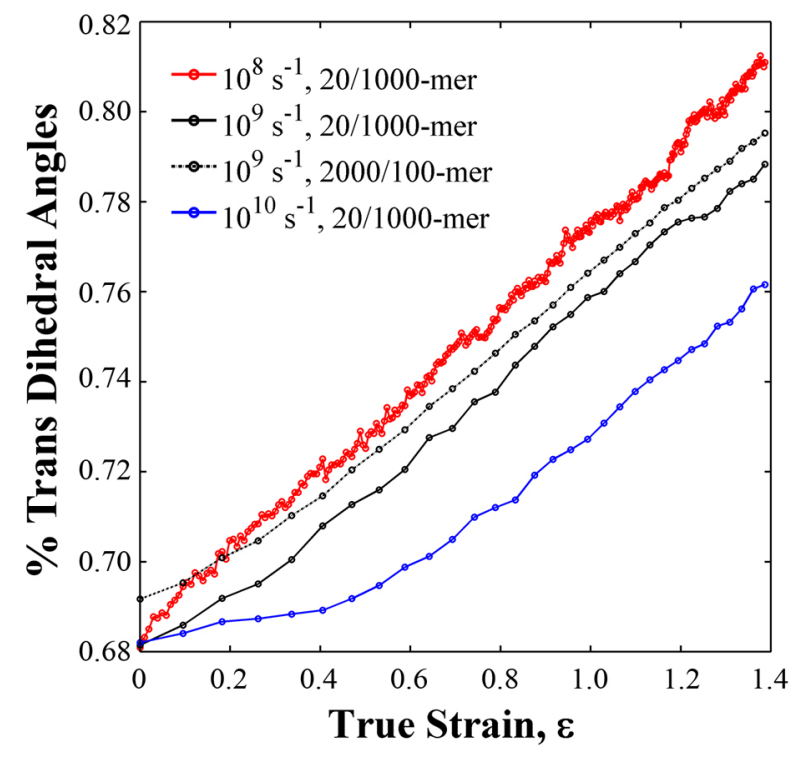

Figure 4: Stress-strain response at $250 \mathrm{~K}$ for different strain rates.

very similar behavior to the 20 1000-mer chain system, when excluding the initially higher \%trans conformations in the former system.

The chain entanglement evolution is also important for deformation of polymer systems. In general, fracture of many polymer materials is expected to occur due to chain disentanglement rather than chain scission, because of the strong carbon-carbon bonds in the polymer backbone. Here, we have used the geometric technique of Yashiro et al. [8] to calculate the chain entanglement. This technique first creates two vectors which originate from each atom to neighboring atoms that are separated by 10 atoms on the same chain, i.e., one vector from atom $i$ with atom $i-10$ and one vector from atom $i$ to atom $i+10$. The angle between these two vectors is calculated for each applicable atom and a threshold parameter of $90^{\circ}$ is used to specify if the atom is classified as entangled or not. The atoms classified as entangled via this technique are considered to be constrained by neighboring chains. Here, we have divided the number of 'entangled' atoms by the total number of atoms to give a normalized entanglement parameter. This entanglement parameter actually represents the percent of entangled atoms and is assumed to be correlated to the entanglement density.

Figure 5 shows the evolution of the entanglement parameter as a function of strain for three different strain rates and two chain lengths at $250 \mathrm{~K}$. Notice that for the initial stages of deformation, the entanglement parameter 


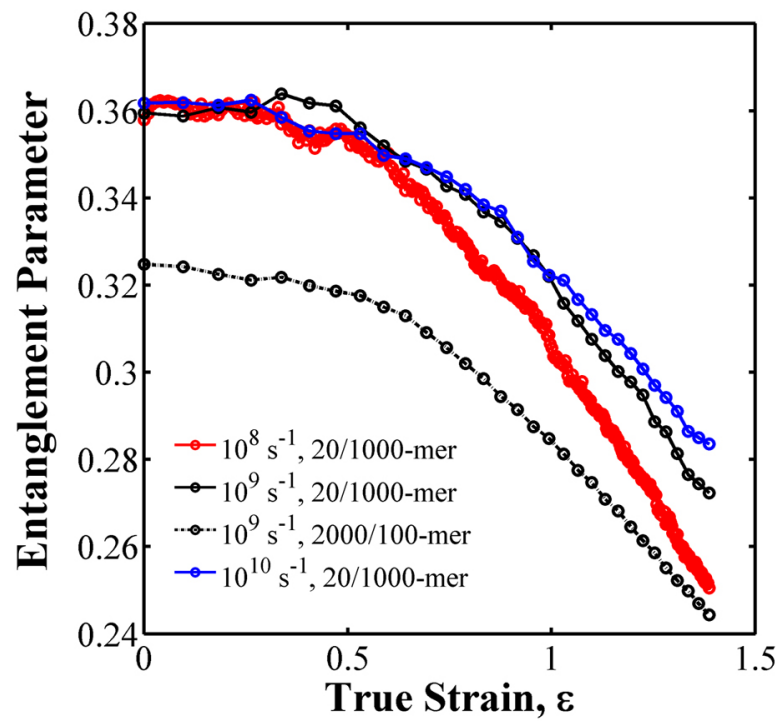

Figure 5: Stress-strain response at $250 \mathrm{~K}$ for different strain rates.

remains somewhat constant with only a slight decrease. At higher strains, indicative of the strain hardening region, the entanglement parameter decreases in a linear fashion. As the strain rate is decreased, the entanglement parameter decreases at a faster rate in the strain hardening region. This is as expected; the lower strain rate will allow more time for chains to disentangle as a way to accommodate the deformation. A similar trend is observed for the shorter chain length system as well, although the entanglement parameter starts at a lower value. This is as expected as well; as the chain length decreases, the tendency should be for the chains to become more difficult to entangle.

\section{Boundary conditions}

The boundary conditions used in atomistic simulations of deformation in material systems needs to be further investigated for polymeric systems. For instance, in the present simulations, the total energy decreases at large strains. However, the uniaxial stress increases over the same strains, implying that additional work is being done to the system (which should result in an increase in internal energy). These responses seem to be contradictory. However, since the thermostat for the NPT boundary conditions is maintaining the temperature of the system through adjusting the atom velocities, the additional work generated in the strain hardening region is being dissipated at a rapid rate through the Nose-Hoover ther- mostat. Currently, it is not known how removing the thermostat or adjusting the rate of heat dissipation in atomistic simulations will impact the energy distribution at higher strains. Future work will explore how the rate of heat dissipation impacts these considerations at the molecular scale.

\section{Acknowledgments}

This work was performed at the Center for Advanced Vehicular Systems (CAVS) at Mississippi State University. The authors would like to acknowledge funding from the Department of Energy.

\section{References}

[1] S.L. Mayo, B.D. Olafson, W.A. Goddard, "DREIDING: A generic force field for molecular simulations," Journal of Physical Chemistry, 94 (1990) 8897.

[2] S. Plimpton, "Fast parallel algorithms for shortrange molecular dynamics," Journal of Computational Physics, 117 (1995) 1.

[3] S.J. Nose, "A unified formulation of the constant temperature molecular dynamics methods," Chemical Physics, 81 (1984) 511.

[4] W.G. Hoover, "Canonical dynamics: Equilibrium phase-space distributions," Physical Review A, 31 (1985) 1695.

[5] K. Binder, Monte Carlo and molecular dynamics simulations in polymer science (New York, NY: Oxford University Press, 1995).

[6] S. Melchionna, G. Ciccottia, B.L. Holian, "Hoover NPT dynamics for systems varying in shape and size," Molecular Physics, 78 (1993) 533.

[7] D. Hossain, M.A. Tschopp, D.K. Ward, J.L. Bouvard, P. Wang, M.F. Horstemeyer, "Molecular dynamics simulations of deformation mechanisms of amorphous polyethylene," Polymer (2010) doi:10.1016/j.polymer.2010.10.009.

[8] K. Yashiro, T. Ito, Y. Tomita, "Molecular dynamics simulation of deformation behavior in amorphous polymer: nucleation of chain entanglements and network structure under uniaxial tension," International Journal of Mechanical Sciences 45 (2003) 1863. 